\title{
Mathematical Modeling of the Influence of the Wind Field Structure in the Atmosphere on the Cloud Formation Processes
}

\author{
Boris A. Ashabokov1,2, Lyudmila M. Fedchenko', Alexander V. Shapovalov, \\ Khazhbara M. Kalov ${ }^{1}$, Ruslan Kh. Kalov ${ }^{1}$, Alla A. Tashilova1, Vitaly A. Shapovalov ${ }^{1}$ \\ ${ }^{1}$ High-Mountain Geophysical Institute, Nalchik, Russia \\ ${ }^{2}$ Institute of Computer Science and Problems of Regional Management, Kabardino-Balkarian Research Center, \\ Russian Academy of Sciences, Nalchik, Russia \\ Email: tashilovaa@mail.ru
}

How to cite this paper: Ashabokov, B.A., Fedchenko, L.M., Shapovalov, A.V., Kalov, K.M., Kalov, R.K., Tashilova, A.A. and Shapovalov, V.A. (2018) Mathematical Modeling of the Influence of the Wind Field Structure in the Atmosphere on the Cloud Formation Processes. Atmospheric and Climate Sciences, 8, 84-96.

https://doi.org/10.4236/acs.2018.81006

Received: December 14, 2017

Accepted: January 14, 2018

Published: January 17, 2018

Copyright ( 2018 by authors and Scientific Research Publishing Inc. This work is licensed under the Creative Commons Attribution International License (CC BY 4.0).

http://creativecommons.org/licenses/by/4.0/

(c) (i) Open Access

\begin{abstract}
The state of the physics of convective clouds and cloud seeding is discussed briefly. It is noted that at the present time there is a transition from the stage of investigation of "elementary" processes in the clouds to the stage of studying the formation of macro- and microstructural characteristics of clouds as a whole, taking into account their system properties. The main directions of the development of cloud physics at the upcoming stage of its development are discussed. The paper points out that one of these areas is the determination of the structure-forming factors for the clouds and the study of their influence on their formation and evolution. It is noted that one of such factors is the interaction of clouds with their surrounding atmosphere, and the main method of studying its role in the processes of cloud formation is mathematical modeling. A three-dimensional nonstationary model of convective clouds is presented with a detailed account of the processes of thermohydrodynamics and microphysics, which is used for research. The results of modeling the influence of the wind field structure in the atmosphere on the formation and evolution of clouds are presented. It is shown that the dynamic characteristics of the atmosphere have a significant effect on the formation of macro- and microstructural characteristics of convective clouds: the more complex the structure of the wind field in the atmosphere (i.e., the more intense the interaction of the atmosphere and the cloud), the less powerful the clouds are formed.
\end{abstract}

\section{Keywords}

Wind in the Atmosphere, Wind Field Structure, Interaction with Clouds, Modeling, System Properties of Clouds, Interaction of Clouds with the 
Atmosphere, Three-Dimensional Cloud Model

\section{Introduction}

The present period of time is a transition period for cloud physics and cloud seeding: a gradual transition from the stage of studying the "elementary" processes in the clouds to the stage of studying the formation and evolution of clouds as a whole, taking into account their system properties [1] [2]. Obviously, such a transition is natural, because cloud physics is not limited to the study of individual processes in the clouds, and there are still many factors affecting the formation and evolution of clouds and requiring study. It should also be noted that the transition of cloud physics to the next stage of evolution will take a certain period of time. This is due to the fact that, on the one hand, such "elementary" processes, whose role in the formation and evolution of clouds are great, remained unexplored or insufficiently studied, and, on the other hand, it is necessary to formulate research directions at the next stage of the evolution of cloud physics, the tasks of these directions, develop methodologies and methods for solving these problems. Taking into account that convective clouds belong to complex physical systems, one of the directions of research of a new stage of its evolution is the determination of the main structure-forming factors for clouds and the study of their influence on the formation of their macro- and microstructural characteristics. Such factors, as noted in [1] [2], are the interaction of clouds with their surrounding atmosphere and the interaction of processes in the clouds (the properties of hierarchy and emergence of systems) [3] [4].

In this paper, we present some results of studies on the effect of the structure of the wind field in the atmosphere on the formation of macro- and microstructural characteristics of convective clouds. It is one of the most important mechanisms of interaction of clouds with their surrounding atmosphere.

Note that this problem has long been in the field of view of researchers. As an example, we can mention papers [5]-[11], which are devoted to the investigation of the possible influence on the processes of cloud formation of various mechanisms of interaction of the atmosphere with clouds. The list of these works can be continued, but without dwelling on this, we note that the authors of these works did not distinguish the belonging of this problem to a new stage in the evolution of cloud physics. In addition, the possibilities of the methods that they used were significantly limited. Therefore, these studies should be carried out from a new perspective and using more effective methods and methodologies.

In this regard, it is important to note that the study of the influence of this factor on the processes of cloud- and precipitation formation processes is possible only on the basis of numerical modeling using full three-dimensional cloud models. In this paper we use for this purpose a three-dimensional numerical model of mixed convective clouds with a detailed account of the processes [1] [2] [12] [13]. The model is effectively used to study various issues related to the 
processes of cloud formation. In particular, on the basis of this model, the formation of the thermodynamic, microstructural and electrical characteristics of convective clouds was studied [14] [15] [16], and the formation of their microstructural characteristics was studied in [17] [18]. Of the works devoted to the study of the role of the interaction of processes in the clouds on the formation of their microstructural characteristics, it can be noted [19]. It presents the results of modeling the influence of deformations in the clouds (formation of the interaction of processes in the clouds) on the formation of microstructural characteristics of hail clouds. We also note that a new method based on the use of the "digital atmosphere" is used to form the input data of the model (initial conditions) [20].

\section{Research Method}

The present work uses a three-dimensional model with detailed account of processes, including electrical ones, to study the effect of the wind field structure in the atmosphere on the formation of macro- and microstructural characteristics of convective clouds.

We present a system of equations describing processes in the clouds to get an idea of the model, and for a more detailed acquaintance with the model, we can recommend the papers [4] [5] [6].

The mathematical model of the convective cloud includes the equations of thermodynamics, microphysics and electrostatics:

$$
\begin{gathered}
\frac{\partial u}{\partial t}+(\boldsymbol{V} \cdot \nabla) u=-\nabla \pi^{\prime}+\Delta^{\prime} u+l v, \\
\frac{\partial v}{\partial t}+(\boldsymbol{V} \cdot \nabla) v=-\nabla \pi^{\prime}+\Delta^{\prime} v-l u, \\
\frac{\partial w}{\partial t}+(\boldsymbol{V} \cdot \nabla) w=-\nabla \pi^{\prime}+\Delta^{\prime} w+g\left(\frac{\theta^{\prime}}{\theta_{0}}+0,61 s^{\prime}-Q_{S}\right),
\end{gathered}
$$

Continuity equation:

$$
\frac{\partial u}{\partial x}+\frac{\partial v}{\partial y}+\frac{\partial w}{\partial z}=\sigma w,
$$

Equations of thermodynamics:

$$
\begin{gathered}
\frac{\partial \theta}{\partial t}+(\boldsymbol{V} \cdot \nabla) \theta=\frac{L_{K}}{c_{p}} \frac{\theta}{T} \frac{\delta M_{K}}{\delta t}+\frac{L_{C}}{c_{p}} \frac{\theta}{T} \frac{\delta M_{C}}{\delta t}+\frac{L_{3}}{c_{p}} \frac{\theta}{T} \frac{\delta M_{3}}{\delta t}+\Delta^{\prime} \theta, \\
\frac{\partial s}{\partial t}+(\boldsymbol{V} \cdot \nabla) s=-\frac{\delta M_{K}}{\delta t}-\frac{\delta M_{C}}{\delta t}+\Delta^{\prime} s,
\end{gathered}
$$

Equations for the distribution functions of droplets, crystals, and frost-fragmentation by mass:

$$
\begin{aligned}
& \frac{\partial f_{1}}{\partial t}+u \frac{\partial f_{1}}{\partial x}+v \frac{\partial f_{1}}{\partial y}+\left(w-V_{1}\right) \frac{\partial f_{1}}{\partial z} \\
& =\left(\frac{\partial f_{1}}{\partial t}\right)_{K}+\left(\frac{\partial f_{1}}{\partial t}\right)_{K \Gamma}+\left(\frac{\partial f_{1}}{\partial t}\right)_{A K}+\left(\frac{\partial f_{1}}{\partial t}\right)_{д P}+\left(\frac{\partial f_{1}}{\partial t}\right)_{3}+\Delta^{\prime} f_{1}+I_{1}
\end{aligned},
$$




$$
\begin{gathered}
\frac{\partial f_{2}}{\partial t}+u \frac{\partial f_{2}}{\partial x}+v \frac{\partial f_{2}}{\partial y}+\left(w-V_{2}\right) \frac{\partial f_{2}}{\partial z} \\
=\left(\frac{\partial f_{2}}{\partial t}\right)_{C}+\left(\frac{\partial f_{2}}{\partial t}\right)_{A K}+\left(\frac{\partial f_{2}}{\partial t}\right)_{3}+\Delta^{\prime} f_{2}+I_{2}+I_{A B} \\
\frac{\partial f_{3}}{\partial t}+u \frac{\partial f_{3}}{\partial x}+v \frac{\partial f_{3}}{\partial y}+\left(w-V_{2}\right) \frac{\partial f_{3}}{\partial z}=\left(\frac{\partial f_{3}}{\partial t}\right)_{3}+\left(\frac{\partial f_{3}}{\partial t}\right)_{A K}+\Delta^{\prime} f_{3},
\end{gathered}
$$

Poisson's equation for the potential of an electrostatic field:

$$
\frac{\partial^{2} U}{\partial x^{2}}+\frac{\partial^{2} U}{\partial y^{2}}+\frac{\partial^{2} U}{\partial z^{2}}=-\frac{\rho_{\mathrm{e}}}{\varepsilon_{0}} .
$$

The initial conditions for Equations (1)-(12) have the following form:

$$
\begin{gathered}
u(\boldsymbol{r}, 0)=u_{0}(\boldsymbol{r}), v(\boldsymbol{r}, 0)=v_{0}(\boldsymbol{r}), w(\boldsymbol{r}, 0)=w_{0}(\boldsymbol{r}), \\
\theta(\boldsymbol{r}, 0)=\theta_{0}(\boldsymbol{r}), s(\boldsymbol{r}, 0)=s_{0}(\boldsymbol{r}), \\
f_{1}(\boldsymbol{r}, m, 0)=f_{2}(\boldsymbol{r}, m, 0)=f_{3}(\boldsymbol{r}, m, 0)=0, \rho_{-}(\boldsymbol{r}, 0)=\rho_{+}(\boldsymbol{r}, 0)=0 .
\end{gathered}
$$

Border conditions:

$$
\begin{gathered}
u(\boldsymbol{r}, t)=u_{0}(\boldsymbol{r}), v(\boldsymbol{r}, t)=v_{0}(\boldsymbol{r}), w(\boldsymbol{r}, t)=w_{0}(\boldsymbol{r}), \\
\theta(\boldsymbol{r}, t)=\theta_{0}(\boldsymbol{r}), s(\boldsymbol{r}, t)=\left.s_{0}(\boldsymbol{r})\right|_{x=0, L_{x} ; y=0, L_{y} ; z=L_{z}} \\
u(\boldsymbol{r}, t)=v(\boldsymbol{r}, t)=w(\boldsymbol{r}, t)=0, \theta(\boldsymbol{r}, t)=\theta_{0}(\boldsymbol{r}), s(\boldsymbol{r}, t)=\left.s_{0}(\boldsymbol{r})\right|_{z=0} \\
f_{1}(\boldsymbol{r}, m, t)=f_{2}(\boldsymbol{r}, m, t)=f_{3}(\boldsymbol{r}, m, t)=\left.0\right|_{x=0, L_{x} ; y=0, L_{y} ; z=L_{z}} \\
\frac{\partial f_{1}(\boldsymbol{r}, m, t)}{\partial z}=\frac{\partial f_{2}(\boldsymbol{r}, m, t)}{\partial z}=\frac{\partial f_{3}(\boldsymbol{r}, m, t)}{\partial z}=\left.0\right|_{z=0} \\
\frac{\partial U(\boldsymbol{r}, t)}{\partial x}=\left.0\right|_{x=0, L_{x}}, \frac{\partial U(\boldsymbol{r}, t)}{\partial y}=\left.0\right|_{y=0, L_{y}}, \frac{\partial U(\boldsymbol{r}, t)}{\partial z}=\left.0\right|_{z=L_{z}}, U(\boldsymbol{r}, t)=\left.0\right|_{z=0}
\end{gathered}
$$

The system of equations is applied for the space-time domain:

$$
0 \leq x \leq L_{x}, 0 \leq y \leq L_{y}, 0 \leq z \leq L_{z}, 0 \leq m<\infty, t>0 .
$$

The following notation is used:

$$
(\boldsymbol{V} \cdot \nabla) \equiv u \frac{\partial}{\partial x}+v \frac{\partial}{\partial y}+w \frac{\partial}{\partial z}, \Delta^{\prime}=\frac{\partial}{\partial x} K \frac{\partial}{\partial x}+\frac{\partial}{\partial y} K \frac{\partial}{\partial y}+\frac{\partial}{\partial z} K \frac{\partial}{\partial z},
$$

$\boldsymbol{r}=\{x, y, z\}$-coordinate vector, $\boldsymbol{V}=\{u, v, w\}$-velocity vector, $u(\boldsymbol{r}), v(\boldsymbol{r})$, $w(\boldsymbol{r})$-velocity vector components; $l$-inertial force parameter; $\theta(\boldsymbol{r})$-potential temperature; $\pi(\boldsymbol{r})=c_{p} \bar{\theta}(P(z) / 1000)^{R / C_{p}}$-dimensionless pressure; $\bar{\theta}$-average potential temperature; $R$-gas constant; $s(\boldsymbol{r})$-specific air humidity; $Q_{S}(\boldsymbol{r})$-the total ratio of the mixture of liquid and solid phases in the cloud; $\sigma(z)$-a parameter that takes into account the change in air density with altitude; $P(z)$ и $T(\boldsymbol{r})$-respectively, pressure and temperature; $c_{p}$-heat capacity of air at constant pressure; $L_{K}, L_{C}, L_{3}$-respectively, the specific heat of condensation, sublimation and freezing; $\pi^{\prime}(\boldsymbol{r}), \theta^{\prime}(\boldsymbol{r}), s^{\prime}(\boldsymbol{r})$-deviations of dimensionless pressure, potential temperature and specific humidity from their background values in the 
ambient atmosphere $\pi_{0}(\boldsymbol{r}), \theta_{0}(\boldsymbol{r}), s_{0}(\boldsymbol{r}) ; \frac{\delta M_{K}}{\delta t}, \frac{\delta M_{C}}{\delta t}$-changes in specific humidity due to the diffusion of steam into droplets and crystals; $\frac{\delta M_{3}}{\delta t}$-the mass of the dropping water that freezes per unit time in a unit volume of air; $K(\boldsymbol{r})$ -turbulent diffusion coefficient. $V_{1}(m), V_{2}(m)$-steady rate of fall of liquid and solid particles; $\left(\frac{\partial f_{1}}{\partial t}\right)_{K},\left(\frac{\partial f_{1}}{\partial t}\right)_{K \Gamma},\left(\frac{\partial f_{1}}{\partial t}\right)_{A K},\left(\frac{\partial f_{1}}{\partial t}\right)_{\not P},\left(\frac{\partial f_{1}}{\partial t}\right)_{3}$-changes in the distribution function of droplets due to microphysical condensation processes, coagulation of droplets, accretion of droplets and crystals, crushing and freezing, respectively; $\left(\frac{\partial f_{2}}{\partial t}\right)_{C},\left(\frac{\partial f_{2}}{\partial t}\right)_{A K},\left(\frac{\partial f_{2}}{\partial t}\right)_{3}$-changes in the distribution function of crystals due to sublimation, accretion and freezing of droplets; $\left(\frac{\partial f_{3}}{\partial t}\right)_{3}$, $\left(\frac{\partial f_{3}}{\partial t}\right)_{A K}$-changes in the distribution function $f_{3}(\boldsymbol{r}, m, t)$ due to the formation of fragments during spontaneous freezing of supercooled cloud droplets and their accretion with crystals; $I_{1}$ and $I_{2}$-sources of droplets and crystals; $I_{A B}$ -source of artificial crystals under cloud seeding; $\varepsilon_{0}$-dielectric constant of vacuum.

The boundaries of the spatial domain are denoted by $0, L_{x}, 0, L_{y}$ и $0, L_{z}$ The remaining notations are given in the works [4] [5] [6].

Powerful convective clouds observed in the North Caucasus on 02.09.2010 and 07.06.2012 and accompanied by the fall of hail were selected for the study. Calculations were made of the formation and evolution of clouds for various wind field structures in the atmosphere, which included the actual structure constructed from the aerological sounding data (the Mineralnye Vody Airport, North Caucasus, Russia) of the atmosphere on the days indicated, and the model wind structures constructed using the same data but modified as planned authors. The remaining parameters of the atmosphere remained unchanged during the calculations. In Figure 1, the real (left) and model (right) wind field structures in the atmosphere are given as an example (02.06.2010). The difference between the real and model field structures, as you can see, is that in the second case, at all levels, the wind velocity vector in the atmosphere is directed along the axis OX.

Comparative analysis of the characteristics of the model clouds (i.e. based on the calculation results) to study the effect of the wind field structure on the processes of cloud- and precipitation formation processes at the same time instants was carried out: the characteristics of the velocity fields of vertical air movements in the cloud and near cloudiness, radar reflectivity, as well as the maximum values of cloud parameters.

\section{Results of Calculations}

Let us dwell on the results of calculations of the cloud corresponding to the real structure of the wind field in the atmosphere. In the Figure 2 and Figure 3 


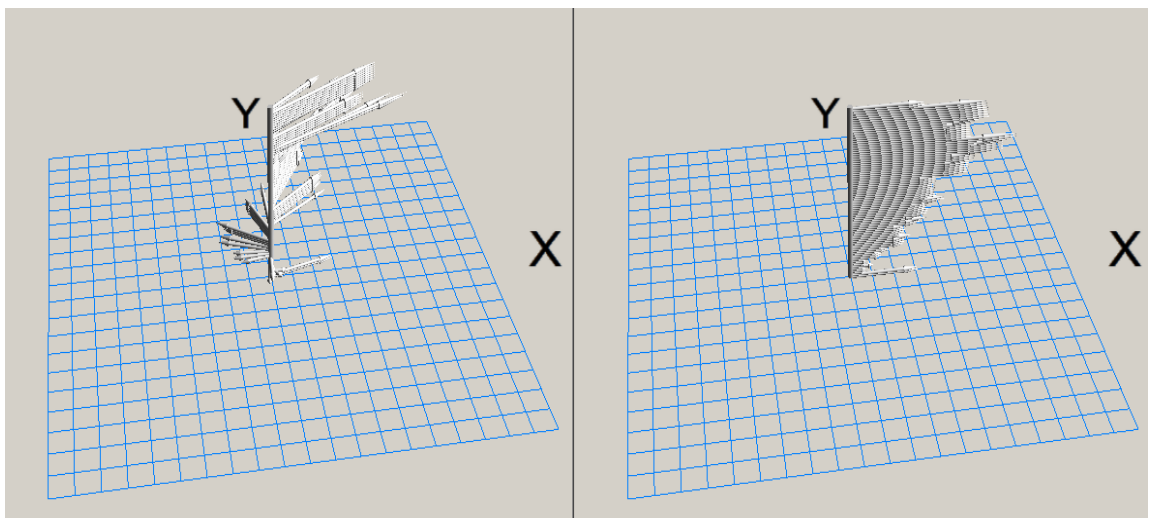

Figure 1. Real (left) and model (right) structure of the wind field in the atmosphere, used for calculations.

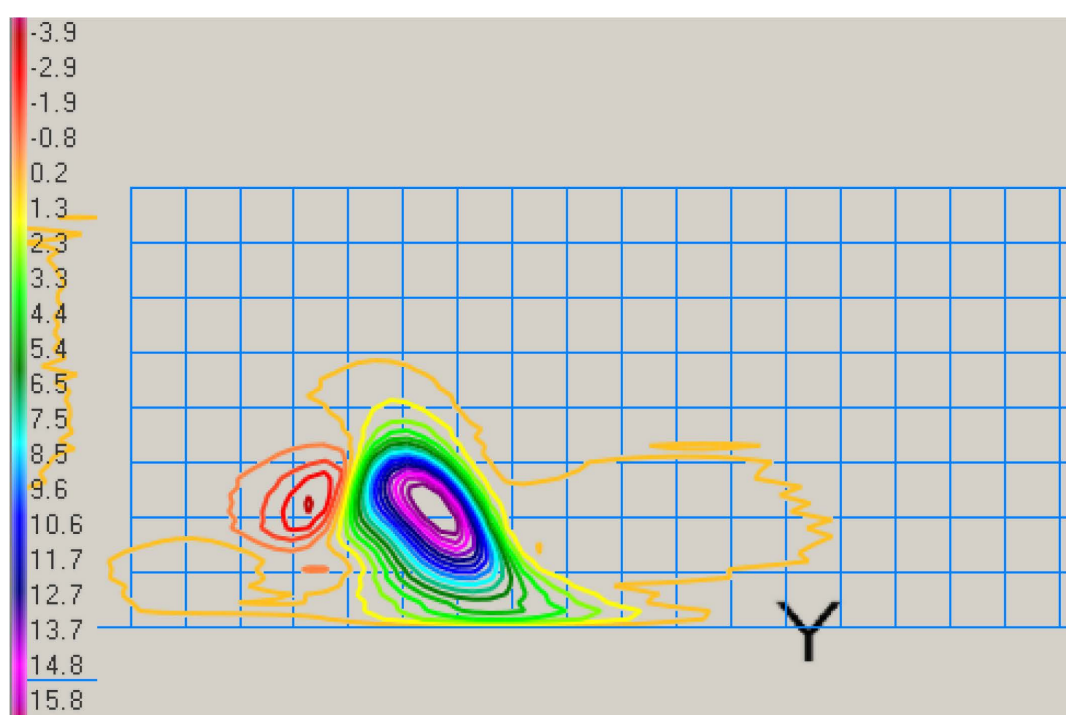

Figure 2. Isolines of upflow velocity in the vertical plane (20th min).

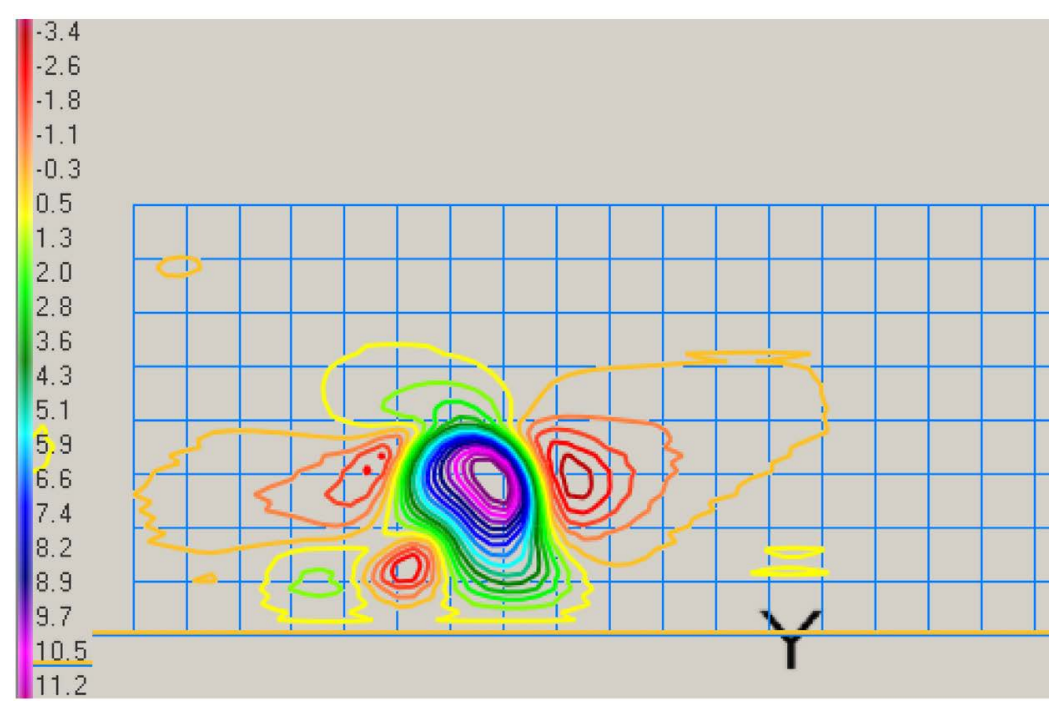

Figure 3. Isolines of different values of the upflow velocity in the vertical plane (30th $\min )$. 
isolines of vertical velocities in the cloud and near cloud are shown in a vertical plane passing through the center of the cloud along the axis OX. The figures correspond to 20 and $30 \mathrm{~min}$ of the evolution of the cloud. It can be noted that the velocity field of the ascending and descending air currents has a rather complex structure, and, as can be seen in the figures, over time it changes noticeably and becomes more complex.

At the same time, the shape of the zone of upflows in the cloud and its inclination to the OX axis change more slowly with time. As for the vertical movements of air around the cloud, the figure shows that the structure of the velocity field of vertical air currents has undergone significant changes. Firstly, in the course of the evolution of the cloud, the area covered by the ascending and descending movements of the air expanded significantly, and secondly, the velocity field structure of these movements became noticeably more complex. As for the maximum values of the velocity of vertical airflows in the cloud, they remain relatively small $-17.9 \mathrm{~m} / \mathrm{s}$ for 20 th $\mathrm{min}$. and $12.8 \mathrm{~m} / \mathrm{s}$ for 30 th $\mathrm{min}$ of the cloud evolution, i.e. there is a slight decrease in the value of this parameter. Figure 4 and Figure 5 show the isolines of the rate of ascending and descending air movements in and around the cloud at the same time in the same vertical plane passing through the center of the cloud along the axis OX. But calculations were made for the model structure of the wind field in the atmosphere, i.e. for the case when the direction of the wind velocity vector at all levels coincides with the direction of the axis OX. In dark colors, the isosurfaces corresponding to the velocities of the ascending currents $\mathrm{W}=35.0$ and $34.1 \mathrm{~m} / \mathrm{s}$ are identified in the figures. Comparison of Figure 4 and Figure 5 shows that the structures of the vertical air movement fields at the points in time under consideration differ markedly, and there is also a noticeable increase in the rate of descending air movements. As for the structure of the field of vertical air movements, one can

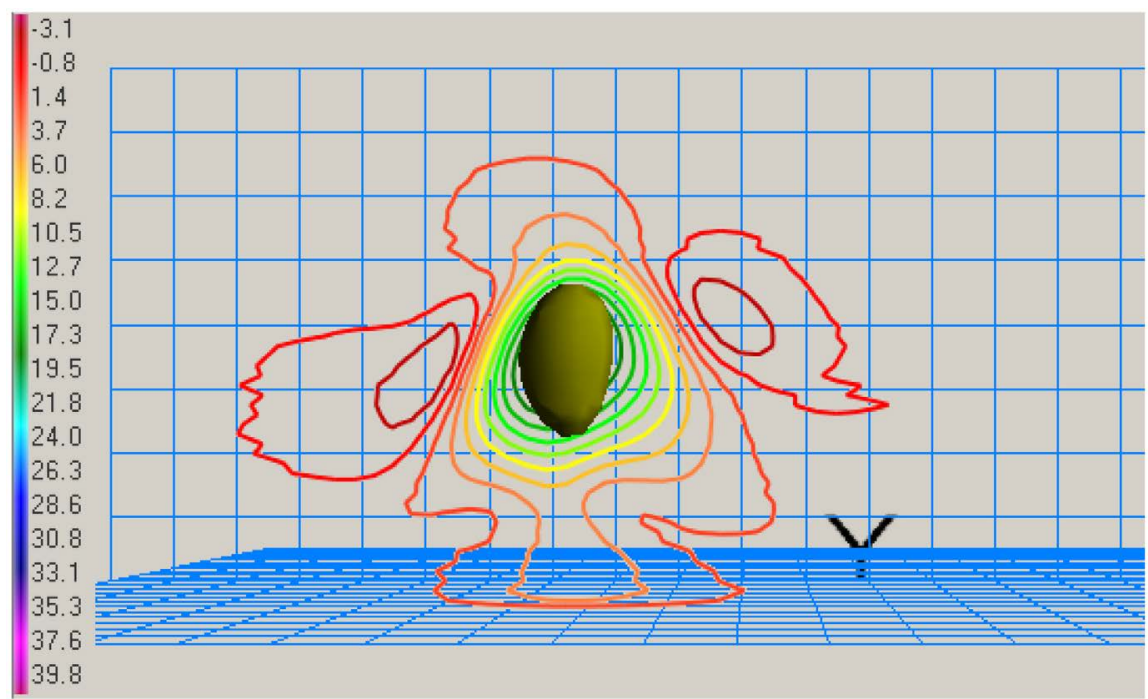

Figure 4. Isolines of different values of the upflow velocity and isosurface $\mathrm{W}=35.0 \mathrm{~m} / \mathrm{s}$ in the vertical plane (20th min). 


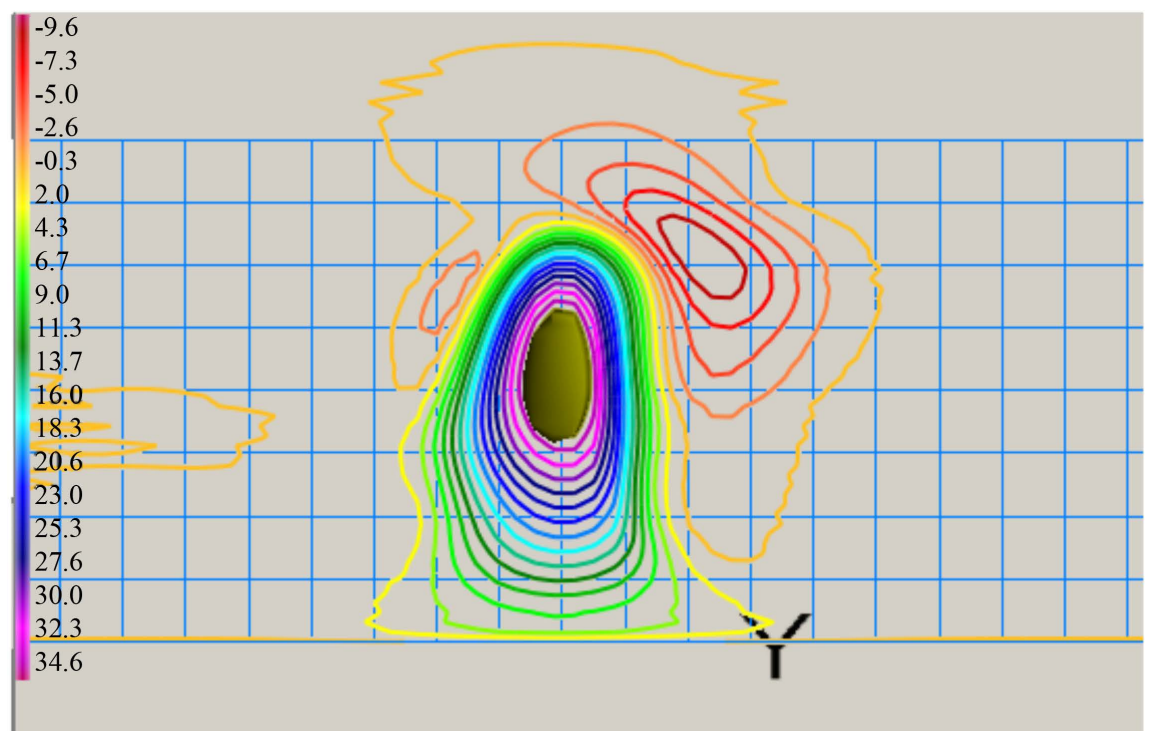

Figure 5. Isolines of vertical stream velocity and isosurface $\mathrm{W}=34.1 \mathrm{~m} / \mathrm{s}$ in the vertical plane (30th $\mathrm{min})$.

notice a significant change in it: the descending air currents in the considered plane under the influence of wind in the atmosphere are localized for 30th min in the upper part of the cloud and on its leeward side.

Comparison of Figure 2 and Figure 3 with Figure 4 and Figure 5 shows that the velocity fields of vertical air movements around the cloud corresponding to different structures of the wind field in the atmosphere differ qualitatively: the structures of vertical air movements in the cloud and near-cloud space corresponding to the real structure of the wind in the atmosphere, are noticeably more complex. As for the maximum rate of ascending airflow in the cloud, attention is drawn to the fact that the values of this parameter at the points of time corresponding to the model structure of the wind field in the atmosphere are significantly higher than its values corresponding to the actual structure of the wind field in the atmosphere.

As the results of calculations show, at all stages of the evolution of the cloud, the influence of the wind field structure in the atmosphere on the zone of ascending currents in the cloud is insignificant, which, in our opinion, is associated with large values of the air velocity in this zone. This zone behaves as an obstacle, which is flowed by horizontal movements of air. Based on the results of calculations, it can be noted that the complication of the structure of the wind field in the atmosphere, in particular, the change in wind speed and direction with altitude is a factor hampering the evolution of convection. This may be due to the fact that in this case the interaction of the cloud and its surrounding atmosphere becomes more intense: the exchange of energy and mass between them takes a more intense character. And the flow from the surrounding atmosphere into a cloud of colder air with less water vapor will hamper its evolution. The values and fields of other parameters of the cloud, in particular, water content and ice content, depend in a significant way on the structure of the wind 
field in the atmosphere. In this case, the water content in the cloud is localized in the region of ascending currents, which agrees with the theory of formation of the liquid-drop fraction in the cloud, and the level of localization of the increased water content may change with time depending on the rate of ascending currents. The region of localization of glaciation is located at higher levels in comparison with the region of localization of droplets. It is important to note that these areas may overlap depending on the stage of the evolution of the cloud. As an example, Figure 6 and Figure 7 show isolines of water content at the 20th min of the cloud evolution, corresponding to the real and model structures of the wind field in the atmosphere.

The figures show that the water-localization zones corresponding to different wind structures in the atmosphere differ qualitatively. Significantly, the values (including the maximum) of this parameter are also different. Comparison of the figures shows that in the case of the model structure of the wind field in the atmosphere, the size of the region of localization of water content in the cloud, as well as its values, is much larger than in the cloud evaluating in the atmosphere with real wind field structure. In Figure 7, it can be seen that the isolines of water content reach the surface of the earth, i.e. in the case of the model structure of the wind field in the atmosphere, precipitation is observed in the cloud. This is explained by the fact that in connection with the more intense airflow in the cloud in the case of the model structure of the wind field in the atmosphere, the formation of precipitation particles also becomes more intense.

$\mathrm{W}=35.0 \mathrm{~m} / \mathrm{s}$ against the background of the isolines of water content in the vertical plane (20th $\mathrm{min}$ ).

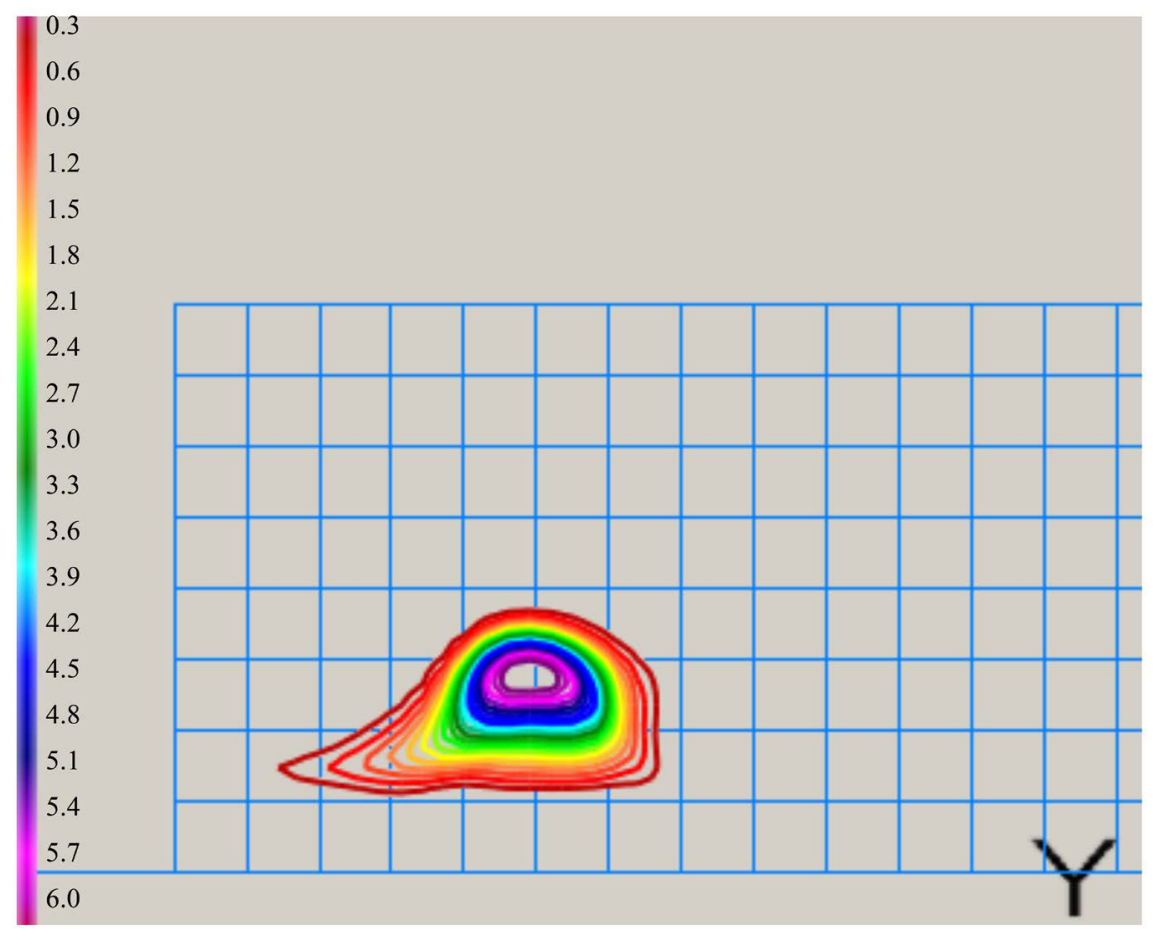

Figure 6. Isolines of water content in the vertical plane (20th min). 


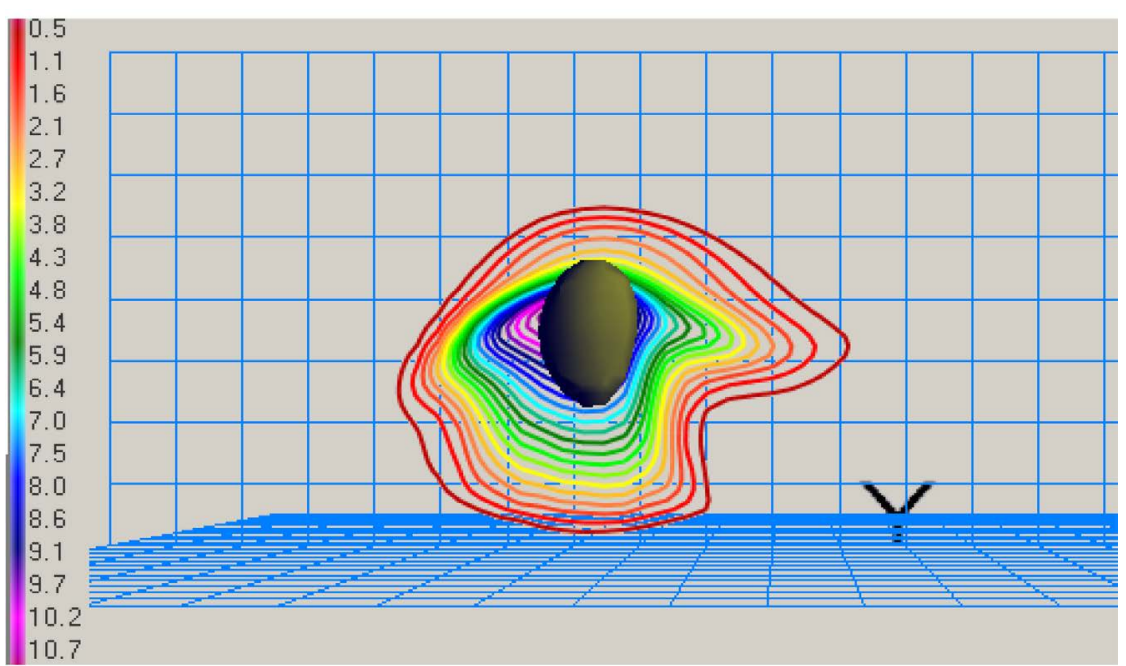

Figure 7. The isosurface of the vertical component of the velocity. $\mathrm{W}=35.0 \mathrm{~m} / \mathrm{s}$ against the background of the isolines of water content in the vertical plane (20th $\mathrm{min}$ ).

The obtained results were supplemented with the results of calculations of the maximum values of the cloud parameters at different instants corresponding to different wind field structures in the atmosphere. These parameter values also give useful information about the effect of the wind field structure in the atmosphere on the formation and evolution of clouds. Table 1 gives the maximum values of the main cloud parameters obtained as a result of calculations. The following designations are used in the table: $\mathrm{W}_{\max }, \mathrm{Q}_{\max }$ and $\mathrm{Z}_{\max }$ are the maximum values of the rate of air upflows and water content in the cloud, and also the reflectivity, $\mathrm{HW}_{\max }, \mathrm{HQ}_{\max }$ and $\mathrm{HZ}_{\max }$ are the levels at which these characteristics are located. It can be seen from the table that the maximum values of the velocity of air up flows $\mathrm{W}_{\max }$ at all stages of the cloud evolution are much larger in the case of the model structure of the wind field in the atmosphere, i.e. in the case when the interaction of the cloud with the surrounding atmosphere is less intense. Accordingly, the heights in which these characteristics are located in the cloud are noticeably higher in this case than in the case of the actual structure of the wind field in the atmosphere. As it is known, the importance of water content plays an important role in the formation and evolution of clouds. It can be seen from the table that the maximum values of this parameter at all times are much larger and located higher in the cloud evaluating in the case of the model structure of the wind field in the atmosphere. In the same way, the maximum glacier values behave in relation to the structure of the wind field in the atmosphere.

Taking into account the maximum water content and altitudes on which they are located in the cloud, it can be noted that in the case when the direction of the wind does not vary in height, the conditions for crystal growth and the formation of hailstones are more favorable. This can be evidenced by the values of maximum reflectivity at different times, corresponding to different structures of the wind field in the atmosphere. In the first case, the maximum value of this 
Table 1. The maximum values of the cloud parameters corresponding to different variants of the wind distribution in the atmosphere.

\begin{tabular}{lcccccc}
\hline \multicolumn{2}{l}{ Parameter } & \multicolumn{2}{c}{ Actual wind field structure in the atmosphere } & \multicolumn{3}{c}{$\begin{array}{c}\text { Model wind field structure } \\
\text { in the atmosphere }\end{array}$} \\
\hline Time, min & 20 & 30 & 40 & 20 & 30 & 40 \\
$\mathrm{~W}_{\max }, \mathrm{m} / \mathrm{s}$ & 17.9 & 12.8 & 7.9 & 44.4 & 39.3 & 39.0 \\
$\mathrm{HW}_{\max }, \mathrm{km}$ & 3.5 & 5.0 & 5.0 & 6.5 & 7.0 & 6.75 \\
$\mathrm{Q}_{\max }, \mathrm{g} / \mathrm{m}^{3}$ & 6.63 & 8.7 & 6.9 & 11.8 & 11.1 & 10.9 \\
$\mathrm{HQ}_{\max }, \mathrm{km}$ & 4.75 & 5.25 & 4.5 & 7.0 & 6.75 & 6.5 \\
$\mathrm{Z}_{\max }, \mathrm{dBZ}$ & 37.6 & 64.7 & 64.3 & 60.0 & 62.8 & 66.6 \\
$\mathrm{HZ}_{\max }, \mathrm{km}$ & 4.25 & 5.25 & 3.75 & 6.5 & 6.0 & 5.5 \\
\hline
\end{tabular}

parameter is reached at the 30th min, and in the second case, an increase in this parameter is observed throughout the considered period of time. In addition, the maximum reflectance value is almost always greater in the case when the direction of the wind does not change with altitude. This indicates that in the case of the model structure of the wind field in the atmosphere, the processes of precipitation formation are more intense.

\section{Conclusions}

A new round of research in cloud physics, connected with the study of the system properties of clouds, is beginning.

The next stage of the development of this scientific direction should be focused on studying the influence of such structure-forming factors, the interaction of processes in the clouds (emergent properties of clouds), and the interaction of clouds with the surrounding atmosphere (hierarchy property). Studies on these and other areas of the development of cloud physics and active effects on them are possible only on the basis of numerical modeling using full three-dimensional and specially constructed cloud models.

The results of numerical experiments have shown that the structure of the wind field in the atmosphere, which is one of the mechanisms of interaction of clouds with their surrounding atmosphere, has a significant influence on the formation and evolution of clouds. These results can be used in forecasting methods of hazardous weather phenomena accompanying convective processes in the atmosphere.

\section{Conflict of Interest}

The authors declare no conflict of interest.

\section{References}

[1] Ashabokov, B.A., Fedchenko, L.M., Tapaskhanov, V.O., Shapovalov, A.V., et al. (2013) The Physics of Hail Clouds and Cloud Seeding: The State and Development Prospects. LLC Printing House, Nalchik, 216. 
[2] Ashabokov, B.A., Fedchenko, L.M., Shapovalov, A.V. and Shapovalov, V.A. (2017) Physics of Clouds and Active Influences on Them. LLC Printing House, Nalchik, 240.

[3] Volkova, V.N. and Denisov, A.A. (2006) Theory of Systems. High School, Moscow, 511.

[4] Mesarovich, M., Mako, D. and Takahara, I.M. (1978) Theory of Hierarchical Multi-Level Systems. Mir, Moscow, 311.

[5] Abshayev, M.T., Goral, G.G. and Malbakhova, N.M. (1987) Forecast of the Hail Process Type. Proceedings of the High-Mountain Geophysical Institute, No. 67, 72-79.

[6] Kovalchuk, A.N. (1969) On the Influence of the Wind Shear on the Fall of Hail. Proceedings of the VGI, Leningrad, No. 14, 39-48.

[7] Malbakhova, N.M. (1990) Interrelation of the Structure of Hail Clouds with the Vertical Structure of the Wind in the Atmosphere. Proceedings of the High-Mountain Geophysical Institute, 80, 99-106.

[8] Newton, C.U. (1964) Hydrodynamic Interaction with the Surrounding Wind Field as One of the Factors of Development of Cumulus Clouds. Dynamics of Cumulus Clouds. Mir, Moscow, 187-201.

[9] Pastushkov, R.S. (1972) Numerical Simulation of the Interaction of Convective Clouds with Their Surrounding Atmosphere. Proceedings of the Central Aerological Observatory $(C A O)$, No. 108, 93-97.

[10] Pastushkov, R.S. (1969) On the Development of Cumulus Clouds in an Atmosphere with Vertical Wind Shear. Meteorology and Hydrology, No. 4, 26-41.

[11] Fedchenko, L.M., Goral, G.G., Belentsova, V.A. and Malbakhova, N.M. (1991) Dangerous Convective Phenomena and Their Forecast in Conditions of Complex Relief. Gidrometeoizdat, Moscow, 425.

[12] Ashabokov, B.A. and Shapovalov, A.V. (2008) Convective Clouds: Numerical Models and Simulation Results in Natural and Active Environments. Publishing house of Kabardino-Balkarian Scientific Center of Russian Academy of Sciences, Nalchik, 257.

[13] Ashabokov, B.A., Fedchenko, L.M., Kupovikh, G.V., Shapovalov, A.V., Skorbezh, N.N. and Shapovalov, V.A. (2012) Model of a Convective Cloud with Allowance for the Influence of Physical Processes on Its Characteristics. News of Higher Schools, North-Caucasian Region, Series: Natural Sciences, Rostov-Don, No. 6, 58-62.

[14] Ashabokov, B.A., Shapovalov, A.V., Kuliev, D.D., Prodan, K.A. and Shapovalov, V.A. (2014) Numerical Simulation of Thermodynamic, Microstructural, and Electric Characteristics of Convective Clouds at the Growth and Mature Stages. Radiophysics and Quantum Electronics, 56, 811-817. https://doi.org/10.1007/s11141-014-9483-Z

[15] Ashabokov, B.A., Kuliev, D.D., Prodan, K.A., Shapovalov, A.V. and Shapovalov, V.A. (2012) Some Results of a Numerical Study of the Formation of Thermodynamic, Microstructural, and Electrical Characteristics of Convective Clouds. Reports of VII All-Russian Conference on Atmospheric Electricity, St. Petersburg, 4-28 September 2012, 31-33.

[16] Kupovykh, G.V., Ashabokov, B.A., Beituganov, M.N., Shapovalov, A.V., Prodan, K.A. and Shapovalov, V.A. (2012) Numerical Simulation of Electrical Characteristics of Convective Clouds. News of Higher Schools, North-Caucasian Region, Series: Natural Sciences, Rostov-Don, No. 6. 65-68. 
[17] Ashabokov, B.A., Shapovalov, V.A., Ezaova, A.G. and Shapovalov, M.A. (2014) Investigation of the Formation of the Ice Phase in High-Power Convective Clouds Based on a Three-Dimensional Numerical Model. Natural and Technical Sciences, No. 5, 78-83.

[18] Shapovalov, A.V., Shapovalov, V.A, Ezaova, A.G. and Prodan, K.A. (2013) Modelling of the Spectra of Particles in Convective Clouds with Mixed Phase Composition and Radiative Properties. News of Kabardino-Balkarian Scientific Center of Russian Academy of Sciences, Nalchik, No. 5, 63-72.

[19] Ashabokov, B.A., Fedchenko, L.M., Shapovalov, A.V. and Shoranov, R.A. (1994) Numerical Studies of the Formation and Growth of Hail with Natural Development of the Cloud and Active Influence. Meteorology and Hydrology, No. 1, 41-48.

[20] Ashabokov, B.A., Kagermazov, A.K., Shapovalov, A.V. and Shapovalov, V.A. (2016) About One Approach to Formation of Starting Conditions at Simulation of Convective Clouds. Works of Main Geophysical Observatory (MGO), St-Petersburg, No. 582, 159-173. 